Recepción: 13 / 08 / 2018

Aceptación: 09 / 10 / 2018

Publicación: 03 / 12 / 2018

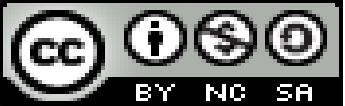

Ciencias económicas y empresariales

Artículo de investigación

\title{
Diseño del plan estratégico para la Cooperativa de Transporte Urbano San Jacinto S.A.
}

Design of the strategic plan for the Urban Transport Cooperative San Jacinto

\author{
S.A.
}

\section{Desenho do plano estratégico para a Cooperativa de Transportes Urbanos San Jacinto S.A.}

\author{
Gustavo R. Castillo-Ruano ${ }^{\mathrm{I}}$ \\ g.castillo.r@hotmail.com \\ Sharon I. Castillo-Valencia II \\ sharonc.valencia@hotmail.com \\ Santiago J. Ludeña-Yaguache ${ }^{\text {III }}$ \\ santiagoludenay@gmail.com
}

Correspondencia: g.castillo.r@hotmail.com

\footnotetext{
I Magíster en Administración de Empresas Mención en Negocios Internacionales, Ingeniero Industrial, Docente de la Universidad Técnica Luis Vargas Torres de Esmeraldas, Esmeraldas, Ecuador.

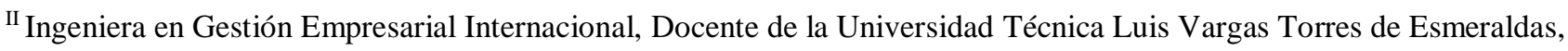
Esmeraldas, Ecuador.

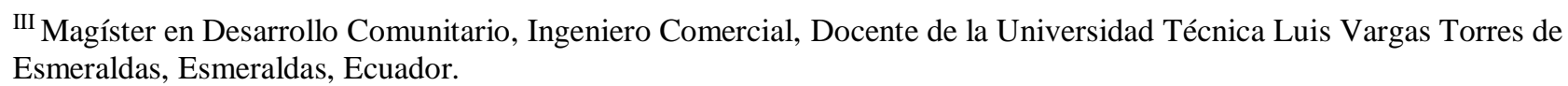




\section{Resumen}

La planeación estratégica, dentro de la gerencia, favorece el desempeño de las organizaciones en un entorno cambiante; brinda la posibilidad de prever situaciones futuras, y tomar decisiones acertadas. El presente trabajo tiene como objetivo proponer el diseño de un Plan Estratégico para mejorar la gestión administrativa de la Cooperativa de Transporte Urbano San Jacinto S.A. Para el desarrollo de este trabajo se recurre al análisis documental que permite la investigación de campo aplicando la observación y la entrevista. Se presenta la misión, la visión, los objetivos, los valores, los factores internos y externos que intervienen en la cooperativa y diferentes matrices que permiten determinar las estrategias para una mejor gestión empresarial.

Palabras clave: plan estratégico; misión visión; objetivos; valores; macroentorno; microentorno; matrices; estrategias; cooperativa.

\section{Abstract}

Strategic planning, within the management, favors the performance of organizations in a changing environment; It offers the possibility of anticipating future situations, and making the right decisions. The objective of this paper is to propose the design of a Strategic Plan to improve the administrative management of the Urban Transport Cooperative San Jacinto S.A. For the development of this work, we resort to the documentary analysis that allows the field investigation applying the observation and the interview. It presents the mission, the vision, the objectives, and the values, the internal and external factors that intervene in the cooperative and different matrices that allow determining the strategies for a better business management.

Key words: strategic plan; mission vision; objectives; values; macroenvironment; microenvironment; matrices; strategies; cooperative.

\section{Resumo}

O planejamento estratégico, dentro da gestão, favorece o desempenho das organizações em um ambiente em mudança; oferece a possibilidade de antecipar situações futuras e tomar as decisões certas. O objetivo deste trabalho é propor a elaboração de um Plano Estratégico para melhorar a gestão administrativa da Cooperativa de Transportes Urbanos San Jacinto S.A. Para o desenvolvimento deste trabalho recorremos à análise documental que permite a investigação de 
campo aplicando a observação e a entrevista. Apresenta a missão, a visão, os objetivos, os valores, os fatores internos e externos que intervêm na cooperativa e as diferentes matrizes que permitem determinar as estratégias para uma melhor gestão empresarial.

Palavras chave: plano estratégico; missão visão; objetivos; valores; macroambiente; microambiente; matrizes; estratégias; cooperativa.

\section{Introducción}

La globalización, obliga a las organizaciones a introducirse en nuevos métodos de gestión administrativa, muchas de éstas no cuentan con las directrices necesarias, y la falta de conocimientos en las áreas de mayor desempeño en la organización causa desmotivación en sus miembros y resultados bajos o insuficientes.

La planeación estratégica, dentro de la gerencia, permite identificar variables internas y externas que influyen en el desempeño de las organizaciones en un entorno cambiante; brinda la posibilidad de prever situaciones futuras, y tomar decisiones acertadas. Para las organizaciones los beneficios que trae la planeación estratégica son necesarios en su desempeño y crecimiento, por lo tanto, es fundamental desarrollar ésta apropiada a sus necesidades. Toda empresa, sin importar su tamaño o naturaleza, necesita elaborar un Plan Estratégico.

Para la Cooperativa de Transporte Urbano San Jacinto S.A aplicar un Plan Estratégico, actualmente es de gran importancia pues el hacerlo representará la mejora de la empresa ya que hasta la fecha ésta ha venido trabajando de manera empírica. Mediante la aplicación del Plan Estratégico la cooperativa puede introducir una cultura de mejoramiento continuo, lo que puede generarle una posición más competitiva en el mercado.

Este trabajo tiene como objetivo general presentar el diseño del Plan Estratégico para mejorar la gestión administrativa de la Cooperativa de Transporte Urbano San Jacinto S.A.

Para cumplimentar el objetivo se realiza un estudio teórico-conceptual que muestra diversos aspectos que sirven de base para la investigación de campo, la cual se basó en la observación directa y la entrevista. Esta se centró en la delimitación de misión, visión, objetivos y valores, el 
análisis de los factores interno y externo, la determinación de los cursos de acción, estrategias y procedimientos para la eficiente gestión administrativa en la Cooperativa a partir del desarrollo de diferentes matrices.

\section{Desarrollo}

\section{Misión, Visión y Objetivos}

La misión de una empresa se constituye en su razón de ser (García-Tenorio Ronda \& Pérez Rodríguez, 1996) enfocada en el presente, es decir, es la actividad que justifica lo que el grupo o el individuo está haciendo en un momento dado. La misión de una empresa depende de la actividad que la organización realice, así como del entorno en el que se encuentra y de los recursos de los que dispone.

Somos una empresa que presta el servicio de transporte urbano, que cubre con seriedad y compromiso las rutas establecidas colaborando así con el desarrollo económico de la ciudad.

La visión de una empresa es el objetivo al que la propia empresa espera llegar en un futuro. Se trata de la expectativa ideal de lo que quiere lograr, indicando a dónde se dirige y cómo desea ser en el largo plazo (Peiro Ucha, 2018). Esta debe ser realista, pero puede ser ambiciosa, su función es guiar y motivar al grupo para continuar con el trabajo.

Para el 2022 ser líderes en el servicio de transporte urbano en de la localidad, apoyado en un mejor capital humano y una flota renovada que garantice la seguridad de nuestros usuarios.

Los objetivos son el planteo de una meta o un propósito a alcanzar, y que, de acuerdo al ámbito donde sea utilizado, tiene cierto nivel de complejidad. El objetivo es una de las instancias fundamentales en un proceso de planificación y que se plantean de manera abstracta en ese principio, pero luego, pueden (o no) concretarse en la realidad, según si el proceso de realización ha sido, o no, exitoso.

Objetivo general: Aumentar la participación de la Cooperativa de Transporte Urbano San Jacinto S.A. en el mercado de transporte urbano mediante el direccionamiento de un Plan Estratégico, y de esta manera mejorar el servicio que esta brinda a la comunidad. 
Objetivos específicos: Incrementar el nivel de confianza y seguridad que los usuarios sienten en nuestras unidades. Fortalecer el capital humano de la institución a través de programas de capacitación orientados a la vocación de servicio y desarrollo personal

Adquirir nuevas unidades con el debido financiamiento para dar un buen servicio a los usuarios.

Impulsar los principios de responsabilidad social creando programas de beneficios sociales para socios y empleados; y efectuando inversiones sociales para la comunidad.

Reducir en un $10 \%$ el tiempo que demora cada bus marcando tarjetas.

Mejorar la calidad de atención al cliente que da el personal.

Incorporar nuevos socios a la cooperativa para que aumente el número de flotas y así brindar un mejor servicio a nuestros usuarios. Reducción del costo por mantenimiento y combustible.

\section{Políticas}

Las políticas empresariales son todas aquellas directrices a las que la empresa decide acogerse, de tal forma que dichas directivas e ideas sean las que motiven las normas generales de actuación de la empresa, determinando así los valores que posee la misma.

Los conductores de la cooperativa serán choferes profesionales quienes tendrán actualizada su licencia respectiva.

Todos los integrantes del recurso humano de la cooperativa demostrarán constantemente vocación de servicio en el desarrollo de sus actividades (buen trato a los usuarios).

Socializar constantemente las leyes, reglamentos, normas y resoluciones relacionadas con las actividades de la cooperativa.

Proporcionar a tiempo y eficientemente la información requerida por los organismos de control competentes.

Usar la vestimenta correspondiente tanto el personal administrativo como el operativo. 


\section{Cadena de valor}

La cadena de valor es un modelo teórico que gráfica y permite describir las actividades de una organización para generar valor al cliente final y a la misma empresa. (Riquelme, 2018)

\section{Actividades primarias}

Logística Interna: Actividades relacionadas con la recepción, almacenamiento y distribución de insumos del producto (manejo de materiales, control de inventarios, devolución a los proveedores, etc.)

Gestión cronológica de buses en espera.

Programación de días para revisión de las unidades.

Designación semanal a cada bus de rutas a cubrir.

Operaciones: Actividades relacionadas con la trasformación de insumos en la forma final del producto (maquinado, mantenimiento de equipo, etc.)

Transportación de pasajeros.

Personal encargado de cobrar dando respeto a los usuarios.

Actividades secundarias

Logística externa: Almacenamiento de los productos terminados y distribución del producto al consumidor.

Llegada y desembarque de pasajeros a su destino.

Comercial / Marketing: Actividades relacionadas con proporcionar un medio por el cual los compradores pueden comprar el producto e inducirlo a hacerlo (publicidad, fuerza de venta, selección del canal, etc.)

Carteles publicitarios en los exteriores de las unidades que pertenecen a la cooperativa. 
Servicio post-venta: Agrupa las actividades destinadas a mantener, realzar el valor del producto, mediante la aplicación de garantías.

Gerente con disponibilidad para recepción de quejas de parte de los usuarios por mala atención, ya sea por estado de los buses o trato del personal.

Infraestructura de la empresa: Consiste en varias actividades, incluyendo la administración general, planeación, finanzas, contabilidad, asuntos legales, etc.

La empresa se encuentra estructurada de la siguiente manera: El gerente (quien despacha las actividades administrativas y de gestión), Secretaria (Encargada de dar apoyo en tramitologías a todo el personal administrativo) Contadora (Encargada de los registros contables y demás actividades financieras) Personal operativo (50 Parejas de chofer y cobradores quienes realizan el transporte urbano. Además de los 55 accionistas que dan apoyo financiero.

Administración de RRHH: Actividades implicadas en la búsqueda, contratación, entrenamiento, desarrollo, etc. de todos los tipos de personal.

El personal administrativo actualmente es fijo, el personal operativo tiende a sufrir cambios o nuevas contrataciones (sobre todo de cobradores) pero estos los realizan el dueño de la unidad, igualmente este se encarga de su entrenamiento inicial.

Desarrollo de tecnologías: Actividades relacionadas con la investigación y desarrollo de la tecnología necesaria para apoyar a las demás.

Actualmente el personal administrativo está en proceso de aprendizaje de nuevas herramientas informáticas y tecnológicas como nuevos CPU con mejores especificaciones.

Aprovisionamiento: Se refiere a la función de comprar insumos utilizados en la cadena de valor, no a insumos comprados en sí.

El gerente de la empresa realiza pequeñas proyecciones de gastos en combustible, además de conseguir varias proformas de precios de los repuestos que se cambian con mayor regularidad. Esto permite proponer acuerdos con los proveedores que permitan minorar los costos. 


\section{Modelo de las 7S}

Este modelo fue creado por la firma consultora estratégica McKinsey en 1980 (Méndez, 2015) y lo componen:

Estilo: Es la cultura de la organización. Normalmente es la cúpula quien debe establecer las bases de los comportamientos y buenas prácticas que marcaran el estilo y la forma de ser de la empresa.

Participativo: Ya que las actividades y toma de decisiones son realizadas de manera conjunta se interesa en el compañerismo que existe en los colaboradores, esto ayuda agilitando la toma de decisiones y tener un buen clima en la empresa.

Personal: Los empleados son la columna vertebral de cualquier organización y uno de sus más importantes activos.

La empresa San Jacinto cuenta con 4 personas para el desarrollo de sus actividades administrativas: Gerente, Contador, Secretaria y Asesor Jurídico

Personal operativo (50 Parejas de conductores y cobradores)

Los trabajadores del área operativa ligados a la empresa, actualmente son reclutados sin tomar como punto importante considerar la preparación académica. En el caso de los choferes, el debido tipo de licencia.

Sistema: Incluye los procesos internos y los sistemas de información que posibilitan el funcionamiento de la empresa

El sistema radica en la coordinación de rutas, que estas estén controladas de manera cronológica respecto al tiempo que cada unidad demora en cada vuelta, lo cual ayude con el buen funcionamiento para lo cual de la empresa y puedan llegar puntual.

Estrategia: Se basa en la manera de organizar y enfocar los recursos, para conseguir los objetivos de la organización. 


\section{Nuestras estrategias son:}

Tener eficacia dentro de la empresa, brindar calidez, seguridad, integridad, excelencia en nuestro servicio para que nuestros clientes quedan satisfechos, mediante la motivación de un buen ambiente laboral a nuestro personal.

Estructura: Es la manera en que se organizan, se relacionan e interactúan las distintas variables $\mathrm{y}$ unidades del negocio

La cooperativa tiene una estructura lineal representada en el Gráfico 1:

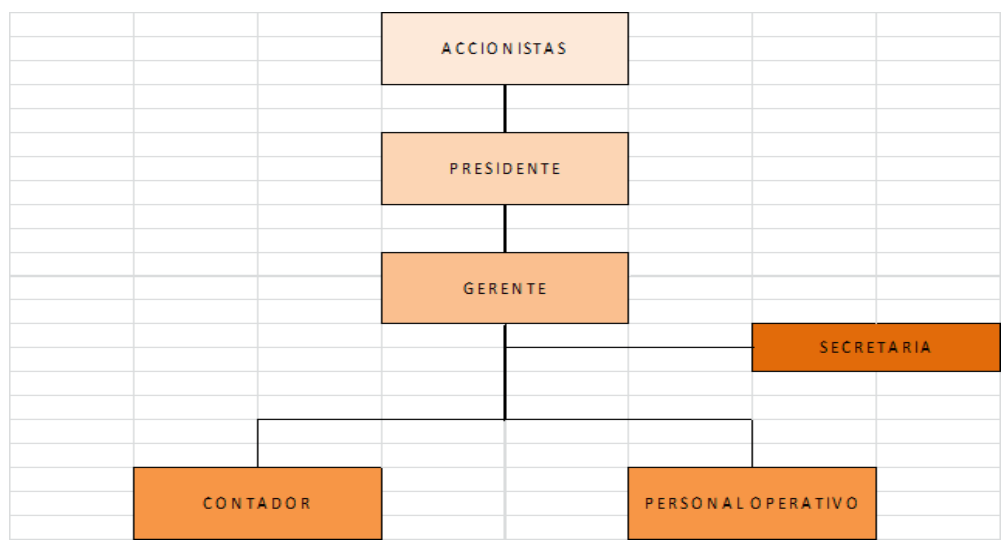

Gráfico 1 Estructura de la Cooperativa

Habilidades: Se refiere a las habilidades y capacidades requeridas por los miembros de la organización.

\section{Las habilidades que poseen los miembros de la cooperativa San Jacinto son:}

Agilidad en manejo de vehículos grandes

Capacidad de reconocer personas en estado etílico.

Habilidad de lidiar con usuarios problemáticos.

Rapidez para sello en la marca tarjetas.

Gerencia con experiencia tomando decisiones importantes. 
Valores: En una organización los valores son el marco del comportamiento que deben tener sus integrantes, y dependen de la naturaleza de la organización (su razón de ser); del propósito para el cual fue creada (sus objetivos); y de su proyección en el futuro (su visión).

\section{Paciencia}

Dado la importancia que tiene la relación que tenemos con nuestros usuarios al momento de atenderlos, consideramos imprescindible demostrar paciencia a cada uno de ellos, permitiendo así que estos sientan comodidad al viajar en nuestras unidades.

\section{Puntualidad}

Cumplir con los horarios establecidos para las diferentes actividades, teniendo en cuenta la importancia que tiene para nuestros usuarios el horario establecido para cada ruta.

\section{Compromiso}

Con el fin de que cada actividad se realice de la mejor manera posible, buscamos que cada miembro de la organización desde la gerencia hasta el nivel operativo se sienta comprometido con sus respectivas actividades.

\section{Respeto}

Dentro y fuera de la empresa es importante mostrar respeto, pues este conyuga el personal de la empresa y da una buena imagen a la comunidad de nuestra organización.

\section{Honestidad}

Buscamos que cada actividad se realice con transparencia total, así los procesos internos en la organización no se ven afectados por recurrentes irregularidades.

\section{Modelo de las 5 fuerzas de Porter}

Las cinco fuerzas Porter es uno de los modelos más famosos que ha elaborado el economista y que dio a conocer en 1979 y en el 2008 (...) (Riquelme Leiva, 2015) 
RIVALIDAD DE LA INDUSTRIA: Rivalidad entre los competidores existentes, interacción entre los competidores de la industria debido a múltiples factores como concentración de empresas para un mismo mercado y existencia de grupos empresariales.

CLIENTES: Poder de negociación de los clientes. Capacidad de los clientes para poner a la empresa bajo presión. Dependencia de unos pocos clientes, grado de dependencia.

PROVEEDORES: Poder de negociación de los proveedores. La capacidad los proveedores para poner a la empresa bajo presión. Dependencia de pocos proveedores, accesibilidad a las materias primas

NUEVOS ENTRANTES: Amenaza de nuevos entrantes. Toda industria atractiva que tiene buenos rendimientos atraerá nuevas empresas

PRODUCTOS SUSTITUTOS: Amenaza de productos sustitutos. Los productos sustitutos están siempre presentes listos para reemplazar los productos de la empresa. Precios bajos de los sustitutos, numero de sustitutos en el mercado

Existen muchos cambios en el macro entorno que repercutirán significativamente en aspectos relacionados con la empresa, tales como: Incremento de la demanda, Herramientas que facilitarán las actividades diarias en los buses (Conteo de personas) y la capacidad económica que tendrán los dueños de las unidades para compra de repuestos. Por lo cual se deben tomar medidas precautelares para mantener una considerable estabilidad.

Poder de negociación con compradores o clientes: Debido a que la competencia tiene rutas muy similares a la nuestra, los clientes tienen un considerable nivel de negociación que presiona a la cooperativa San Jacinto para mejorar su atención y servicio, convirtiéndose esto un determinante punto para que nos escojan sobre la competencia. Es por esto que la cooperativa realiza reuniones con los choferes y oficiales, destacando el trato al cliente.

Poder de negociación con los proveedores: dado a que, en la localidad, el número de gasolineras es considerable con relación a la cantidad de buses en circulación, existe un elevado nivel de poder de negociación por parte de la cooperativa con los proveedores ya que se puede establecer tratos formales buscando reducción de costos para cuando se realicen los despachos de 
combustibles diarios. La cooperativa siempre es puntual en sus pagos convirtiéndola en cliente potencial para sus proveedores por lo cual no les conviene perder nuestro consumo. Sus principales proveedores son: Carrocerías PICOSA y gasolineras P y S, Petronahuaico y Shell. Amenazas de nuevos entrantes: En la actualidad esta amenaza es considerablemente baja, pues dado que el perímetro que necesita ser cubierto por las cooperativas de transporte urbano en la localidad, actualmente se encuentra cubierto en un $90 \%$ aproximadamente. Y no existe demanda que justifique la creación de una nueva cooperativa.

Amenazas de productos sustitutivos: Esta amenaza es continua, pero se está minimizando, gracias a que cada vez son exhaustivos y rígidos el control de transporte público. Pero no se puede dejar de lado a los taxistas informales que hacen de taxi ruta en algunos sectores del cantón.

Rivalidad entre competidores: Existe una fuerte rivalidad con la cooperativa las Palmas ya que esta fue pionera en este tipo de negocios esta cooperativa cubre con la mayor parte del perímetro de recorrido del cantón.

FACTORES DE MACROENTORNO: El macro entorno está compuesto por todos aquellos factores económicos, tecnológicos, políticos, legales, sociales, culturales y medioambientales que afectan al entorno de la empresa. Representa a todas las fuerzas externas y que no son controlables por la empresa.

Nuevos tratados comerciales.

Nuevos enfoques políticos socialistas.

Nivel de corrupción.

Política de multas.

Leyes antimonopolio.

Políticas de aranceles.

Endeudamiento del país. 
Menor poder adquisitivo de la persona.

Créditos con entidades financieras públicas (CFN-BE).

Aumento de costo de repuestos.

Cultura de la población ecuatoriana.

Mejoramiento en el nivel educativo.

Aumento de la población nacional.

Nivel de delincuencia.

Aumento de extranjero en la región.

Reducción de empleos en instituciones públicas.

Aumento en la tasa de desempleo.

Narcotráfico.

Nuevos sistemas contable.

Mejoramiento en métodos de rastreo.

Desarrollo de nuevas herramientas tecnológicas para conteo e pasajeros.

Nuevos sistemas automáticos de protección para chofer aplicables los buses (airbag)

El MICROENTORNO está referido a aquellos factores que son en parte controlables por parte de la empresa.

PROVEEDORES:

*Gasolineras: P y S, Petronahuaico, Shell

*Carrocerías PICOSA. 


\section{COMPETIDORES:}

\section{*Cooperativa de Transporte Urbano Las Piedras}

*Taxis informales

\section{CLIENTE:}

\section{Ciudadanía en general}

El marco de la formulación está compuesto por una matriz de evaluación del factor interno (EFI), una matriz de evaluación del factor externo (EFE) y una matriz del perfil competitivo. (Murillo \& Chasiluisa, 2012)

La matriz EFI es una herramienta para la formulación de la estrategia resume y evalúa las fortalezas y las debilidades principales en las áreas funcionales de una empresa, al igual que proporciona una base para identificar y evaluar las relaciones entre estas áreas.

La matriz EFE permite a los estrategas resumir y evaluar la información económica, social, cultural, demográfica, ambiental, política gubernamental, legal, tecnológica y competitiva. La matriz EFE se desarrolla en cinco pasos.

La matriz del perfil competitivo es una herramienta que permite a la empresa tener bien identificados a los competidores más cercanos. Aquí es muy importante tener cuidado en elegir los factores clave con que nos vamos a comparar, así como a la asignación de ponderaciones, ya que es de una forma subjetiva, y, por tanto, es primordial un conocimiento de la empresa sustentado en estudios documentales; los resultados pueden analizarse de diversos ángulos y de diferentes puntos de vista. 
Diseño del plan estratégico para la Cooperativa de Transporte Urbano San Jacinto S.A.

\begin{tabular}{|c|c|c|c|c|}
\hline \multicolumn{5}{|c|}{ MATRIZ DE EVALUACIÓN DE LOS FACTORES INTERNOS } \\
\hline FACTORES & VALOR & PESO & PUNTUACION & TOTAL \\
\hline \multicolumn{5}{|l|}{ FORTALEZAS } \\
\hline $\begin{array}{l}\text { F1. Funcionamiento administrativo en oficinas } \\
\text { propias }\end{array}$ & 90 & 0,07 & 3 & 0,21 \\
\hline $\begin{array}{l}\text { F2. Personal operativo con licencia y demás } \\
\text { documentación en regla }\end{array}$ & 80 & 0,06 & 3 & 0,19 \\
\hline F3. $\quad$ Cooperativa legalmente constituida & 70 & 0,06 & 3 & 0,17 \\
\hline $\begin{array}{l}\text { F4. Personal con predisposición para } \\
\text { cambios y mejoras }\end{array}$ & 80 & 0,06 & 4 & 0,25 \\
\hline F5. Buena relación con proveedores & 90 & 0,07 & 3 & 0,21 \\
\hline $\begin{array}{l}\text { F6. Cantidad de unidades de flota suficientes } \\
\text { para la demanda }\end{array}$ & 80 & 0,06 & 3 & 0,19 \\
\hline $\begin{array}{l}\text { F7. Gerencia con experiencia en este tipo de } \\
\text { negocio. }\end{array}$ & 80 & 0,06 & 4 & 0,25 \\
\hline F8. $\quad$ Buenas relaciones laborales. & 80 & 0,06 & 3 & 0,19 \\
\hline \multicolumn{5}{|l|}{ DEBILIDADES } \\
\hline D1. Ausencia de organigrama estructural. & 70 & 0,06 & 1 & 0,06 \\
\hline $\begin{array}{l}\text { D2. Dirigencia de la cooperativa con } \\
\text { conocimiento administrativolimitado. }\end{array}$ & 80 & 0,06 & 1 & 0,06 \\
\hline $\begin{array}{l}\text { D3. Inexistencia de sistemas de información } \\
\text { empresarial apropiados }\end{array}$ & 80 & 0,06 & 2 & 0,13 \\
\hline D4. Inexistencia de reglamento interno & 80 & 0,06 & 1 & 0,06 \\
\hline D5. Inexistencia de políticas y valores & 70 & 0,06 & 1 & 0,06 \\
\hline $\begin{array}{l}\text { D6. Actuales oficinas administrativas con } \\
\text { espacio reducido }\end{array}$ & 70 & 0,06 & 2 & 0,11 \\
\hline $\begin{array}{l}\text { D7. Sistema de gestión y control de actividades } \\
\text { obsoleto }\end{array}$ & 90 & 0,07 & 2 & 0,14 \\
\hline $\begin{array}{l}\text { D8. Poca coordinación de actividades entre los } \\
\text { trabajadores }\end{array}$ & 80 & 0,06 & 1 & 0,06 \\
\hline TOTAL & 1270 & 1,00 & 37 & 2,34 \\
\hline
\end{tabular}

Tabla 1 Matriz de Evaluación de los Factores Internos.

Mediante la Matriz EFI (Tabla 1) se obtuvo el resultado de 2,34 lo que indica que la empresa es débil internamente y debe enfocarse mucho en la mejora de las debilidades que retrasan el crecimiento de la empresa. 


\begin{tabular}{|c|c|c|c|c|}
\hline \multicolumn{5}{|c|}{ MATRIZ DE EVALUACIÓN DE LOS FACTORES EXTERNOS } \\
\hline FACTORES & VALOR & PESO & PUNTUACIÓN & TOTAL \\
\hline \multicolumn{5}{|l|}{ OPORTUNIDADES } \\
\hline $\begin{array}{l}\text { O1. Financiamiento externo para la renovación } \\
\text { de oficinas administrativas }\end{array}$ & 70 & 0,05 & 4 & 0,22 \\
\hline $\begin{array}{l}\text { O2. Incremento de estudiantes que demandan } \\
\text { transporte urbano }\end{array}$ & 80 & 0,06 & 4 & 0,25 \\
\hline $\begin{array}{l}\text { O3. Desarrollo de nuevas tecnología para el } \\
\text { control de actividades }\end{array}$ & 70 & 0,05 & 4 & 0,22 \\
\hline $\begin{array}{l}\text { O4. Mejoramiento en vías de circulación dentro } \\
\text { del cantón }\end{array}$ & 80 & 0,06 & 4 & 0,25 \\
\hline $\begin{array}{l}\text { O5. Demanda insatisfecha en la ruta } \\
\text { correspondiente a la competencia }\end{array}$ & 90 & 0,07 & 3 & 0,21 \\
\hline O6. Seminarios gratuitos & 90 & 0,07 & 3 & 0,21 \\
\hline $\begin{array}{l}\text { O7. Posibilidad de ampliar el perímetro } \\
\text { permitido para el recorrido. }\end{array}$ & 90 & 0,07 & 3 & 0,21 \\
\hline $\begin{array}{l}\text { O8. Desarrollo de nuevos métodos de control } \\
\text { de recorridos }\end{array}$ & 70 & 0,05 & 4 & 0,22 \\
\hline \multicolumn{5}{|l|}{ AMENAZAS } \\
\hline A1. Posible aumento en el costo de combustible & 90 & 0,07 & 1 & 0,07 \\
\hline A2. Aumento de transporte informal & 90 & 0,07 & 2 & 0,14 \\
\hline $\begin{array}{l}\text { A3. Atraso en el desarrollo económico de la } \\
\text { Provincia }\end{array}$ & 90 & 0,07 & 1 & 0,07 \\
\hline $\begin{array}{l}\text { A4. Aumento en el costo de mantenimiento y de } \\
\text { implementos de las unidades. }\end{array}$ & 80 & 0,06 & 2 & 0,12 \\
\hline $\begin{array}{l}\text { A5. Competencia obtiene nuevo acuerdo para } \\
\text { ruta universitaria. }\end{array}$ & 70 & 0,05 & 2 & 0,11 \\
\hline A6. Aumento de la inseguridad social & 80 & 0,06 & 2 & 0,12 \\
\hline $\begin{array}{l}\text { A7. Presentación de nuevas unidades de } \\
\text { transporte en la competencia. }\end{array}$ & 70 & 0,05 & 2 & 0,11 \\
\hline $\begin{array}{l}\text { A8. Constantes cierres de carretera por } \\
\text { reparación de tuberías. }\end{array}$ & 80 & 0,06 & 1 & 0,06 \\
\hline TOTAL & 1290 & 1,00 & 42 & 2,58 \\
\hline
\end{tabular}

Tabla 2 Matriz de Evaluación de Factores Externos.

El resultado de la aplicación MEFE (Tabla 2) es de 2,58 (Ligeramente sobre la media) lo cual indica que la organización tiene oportunidades de mantenerse y crecer, pero relativamente bajas. 
Diseño del plan estratégico para la Cooperativa de Transporte Urbano San Jacinto S.A.

MATRIZ DE PERFIL COMPETITIVO

\begin{tabular}{|c|c|c|c|c|c|c|c|}
\hline \multirow[b]{2}{*}{ FACTORES CLAVES DEL ÉXITO } & \multirow[b]{2}{*}{ PONDERACIÓN } & \multicolumn{2}{|c|}{ Illesca } & \multicolumn{2}{|c|}{ Palmas } & \multicolumn{2}{|c|}{ Taxis Informales } \\
\hline & & CLASIFICACIÓN & $\begin{array}{l}\text { RESULTADO } \\
\text { PONDERADO }\end{array}$ & CLASIFICACIÓN & $\begin{array}{l}\text { RESULTADO } \\
\text { PONDERADO }\end{array}$ & CLASIFICACIÓN & $\begin{array}{l}\text { RESULTADO } \\
\text { PONDERADO }\end{array}$ \\
\hline PARTICIPACIÓN EN EL MERCADO & 0,25 & 3 & 0,75 & 3 & 0,75 & 2 & 0,5 \\
\hline TIEMPO EN EL MERCADO & 0,1 & 2 & 0,2 & 3 & 0,3 & 1 & 0,1 \\
\hline POSICIÓN FINANCIERA & 0,1 & 2 & 0,2 & 3 & 0,3 & 1 & 0,1 \\
\hline TECNOLOGÍA & 0,07 & 2 & 0,14 & 3 & 0,21 & 2 & 0,14 \\
\hline SERVICIO AL CLIENTE & 0,1 & 4 & 0,4 & 4 & 0,4 & 2 & 0,2 \\
\hline CALIDAD DE SERVICIO & 0,1 & 3 & 0,3 & 2 & 0,2 & 2 & 0,2 \\
\hline ADMINISTRACIÓN & 0,1 & 2 & 0,2 & 3 & 0,3 & 1 & 0,1 \\
\hline EXPANSIÓN & 0,09 & 2 & 0,18 & 3 & 0,27 & 1 & 0,09 \\
\hline TOTAL & 0,91 & & 2,37 & & 2,73 & & 1,43 \\
\hline & 1 & \multicolumn{2}{|c|}{ debilidad principal } & & & & \\
\hline & 2 & \multicolumn{2}{|c|}{ debilidad menor } & & & & \\
\hline & 3 & \multicolumn{2}{|c|}{ fortaleza menor } & & & & \\
\hline & 4 & \multicolumn{2}{|c|}{ fortaleza principal } & & & & \\
\hline
\end{tabular}

Tabla 3 Matriz de Perfil Competitivo

La matriz de PC (Tabla 3) nos indica que la empresa actualmente cuenta con un nivel competitivo bajo en relación con su principal competidor y ligeramente alto en relación con su competidor menos agresivo, lo cual sugiere idear y aplicar estrategias que aumenten la capacidad competitiva de la empresa en el mercado.

La etapa de ajuste se concentra en generar estrategias alternativas viables, alineando factores internos y externos clave. Incluyen la matriz de las amenazas-oportunidades debilidades-fuerzas (FODA), la matriz de la posición estratégica y la evaluación de las acciones (PEEA), la matriz interna-externa (IE) y la matriz de la estrategia principal. Estos instrumentos dependen de la información derivada de la etapa de aportación de información para ajustar las oportunidades y las amenazas externas con las fuerzas y debilidades internas. Ajustar los factores externos e internos críticos determinantes para el éxito, resulta fundamental para generar buenas estrategias alternativas viables. 


\begin{tabular}{|c|c|c|}
\hline & FORTALEZAS & DEBILIDADES \\
\hline & F1. Funcionamiento administrativo en oficinas propias & D1. Ausencia de organigrama estructural. \\
\hline & F2. Personaloperativoconlicenciaydemásdocumentaciónenregla & D2. Dirigencia de la cooperativa con conocimiento administrativo limitado. \\
\hline & F3. Cooperativa legalmente constituida & D3. Inexistencia de sistemas de información empresarial apropiados \\
\hline & F4. Personal con predisposición para cambios y mejoras & D4. Inexistencia de reglamento interno \\
\hline & F5. Buena relación con proveedores & D5. Inexistencia de políticas y valores \\
\hline & F6. Cantidad de unidades de flota suficientes para la demanda & D6. Actuales oficinas administrativas con espacio reducido \\
\hline & F7. Gerencia con experiencia en este tipo de negocio. & D7. Sistema de gestión y control de actividades obsoleto \\
\hline & F8. Buenas relaciones laborales. & D8. Poca coordinación de actividades entre los trabajadores \\
\hline OPORTUNIDADES & ESTRATEGIA F0 & ESTRATEGIA D0 \\
\hline $\begin{array}{l}\text { 01. Financiamiento externo para la renovación de } \\
\text { oficinas administrativas }\end{array}$ & $\begin{array}{l}\text { F4/06. Aprovechar la predisposición del personal para los cambios, organizando } \\
\text { los horarios para que asistan a las capacitaciones gratuitas. }\end{array}$ & $\begin{array}{l}\text { 01/D6 Gracias a la posibilidad de acceder a un credito, se implementa proyecto } \\
\text { de construccion de nuevas y mejores oficinas. }\end{array}$ \\
\hline $\begin{array}{l}\text { O2. Incremento de estudiantes que demandan } \\
\text { transporte urbano }\end{array}$ & $\begin{array}{l}\text { F6/02. Reestructuración de los horarios de la ruta, según la hora de inicioy } \\
\text { culminación de las jornadas estudiantiles. }\end{array}$ & $\begin{array}{l}\text { 03/D7. Aplicación de las nuevas tecnologias aplicables en el control de actividades } \\
\text { que existen, para remplazar los obsoletos que se usan en la empresa. }\end{array}$ \\
\hline $\begin{array}{l}\text { O3. Desarrollo de nuevas tecnología para el } \\
\text { controlde actividades }\end{array}$ & $\begin{array}{l}\text { F7/08. Adquisición de nuevos sistemas de control que serán utilizados por la } \\
\text { gerencia que les permita explotar su experticia, }\end{array}$ & $\begin{array}{l}\text { 08/D2. Apliacion de nuevos metodos de control de recorridos que faciliten el } \\
\text { seguimiento a los encargados de realizar esta actividad }\end{array}$ \\
\hline $\begin{array}{l}\text { 04. Mejoramiento en vías de circulacióndentro } \\
\text { del cantón }\end{array}$ & $\begin{array}{l}\text { F3/07. Aprovechar que la empresa está legalmente constituida para realizar } \\
\text { petición formal de acceso a nuevas rutas. }\end{array}$ & $\begin{array}{l}\text { 06/D8 Agendar fechas donde se realicen seminarios gratuitos con tematicas de } \\
\text { trabajo enequipo. }\end{array}$ \\
\hline $\begin{array}{l}\text { O5. Demanda insatisfecha en la ruta } \\
\text { correspondiente a la competencia }\end{array}$ & & \\
\hline O6. Seminarios gratuitos & & \\
\hline $\begin{array}{l}\text { 07. Posibilidadde ampliarel perímetropermitido } \\
\text { para elrecorrido. }\end{array}$ & & \\
\hline $\begin{array}{l}\text { O8. Desarrollo de nuevos métodos de control } \\
\text { de recorridos }\end{array}$ & & \\
\hline AMENAZAS & ESTRATEGIA FA & ESTRATEGIA DA \\
\hline A1. Posible aumentoen el costo de combustible & $\begin{array}{l}\text { F3/A2 Aprovechando que somos una cooperativa legalmente constituida, } \\
\text { presentar una petición formal para el aumento de controles del transporte } \\
\text { informal, a las autoridades competentes }\end{array}$ & $\begin{array}{l}\text { D4/A4 Adopción de nuevo sistema de control que disminuyapérdidas y permita } \\
\text { disponer de mayor liquides para pagos de mantenimiento. }\end{array}$ \\
\hline A2. Aumento de transporte informal & $\begin{array}{l}\text { F5/A4 Mediante la buena relación con proveedores, solicitar proformas de } \\
\text { precios para escoger los más apropiados. }\end{array}$ & $\begin{array}{l}\text { D8/A8 Implementar sistema de radio entre choferes que mejore su comunicación y } \\
\text { coordinación, para mejorar la capacidad de reacción ante cierre de vías. }\end{array}$ \\
\hline $\begin{array}{l}\text { A3. Atraso en el desarrollo económico de la } \\
\text { Provincia }\end{array}$ & $\begin{array}{l}\text { F7/A6 Mediante la experiencia que tienen los administradores se puede } \\
\text { seleccionar rutas con menor índice de delincuencia. }\end{array}$ & $\begin{array}{l}\text { D6/A3 Ampliar el tamaño de las oficinas que mejore las actividades } \\
\text { administrativas, mejorando la productividad y estabilidad económica de la } \\
\text { empresa }\end{array}$ \\
\hline $\begin{array}{l}\text { A4. Aumento en el costo de mantenimiento y de } \\
\text { implementos delas unidades. }\end{array}$ & F6/A3 Mediante la capacidad de abarcación de territorio por la cantidad de & D2/A5.Programarfechasparaqueladirigenciaacuda aseminariossobrela \\
\hline $\begin{array}{l}\text { A5. Competencia obtiene nuevo acuerdo para ruta } \\
\text { universitaria. }\end{array}$ & $\begin{array}{l}\text { unidadesen latlota, rotacionregularquegenererentabilidadsuticientepara } \\
\text { sustentar laeconomía. }\end{array}$ & $\begin{array}{l}\text { administración que le otorguen habilidades gerenciales, como negociar nuevos } \\
\text { acuerdos en ruta. }\end{array}$ \\
\hline A6. Aumento de la inseguridad social & & \\
\hline $\begin{array}{l}\text { A7. Presentación de nuevas unidades de } \\
\text { transporte en la competencia. }\end{array}$ & & \\
\hline $\begin{array}{l}\text { A8. Constantes cierres de carretera por } \\
\text { reparación de tuberías. }\end{array}$ & & \\
\hline
\end{tabular}

Tabla 4 Matriz Debilidades-Amenazas-Fortalezas-Oportunidades

La matriz FODA (Tabla 4) nos indica que la empresa está en condiciones de aplicar estrategias factibles que le permitan contrarrestar aquellos factores negativos que existen dentro y fuera de la empresa. 
Diseño del plan estratégico para la Cooperativa de Transporte Urbano San Jacinto S.A.

\begin{tabular}{|c|c|c|c|}
\hline \multicolumn{4}{|c|}{ Matriz PEYEA } \\
\hline \multicolumn{2}{|l|}{ Posición estratégica interna } & \multicolumn{2}{|l|}{ Posición estratégica externa } \\
\hline Fuerza financiera (FF) & Valor & Estabilidad del Ambiente (EA) & Valor \\
\hline $\begin{array}{l}\text { Rendimiento de la inversión } \\
\text { El rendimiento por cada una de las } \\
\text { inversiones realizadas en los últimos años } \\
2 \text { ha bajado en } 5 \%\end{array}$ & 3 & $\begin{array}{l}\text { Variabilidad de la demanda } \\
\text { Ha habido una constante fluctuación negativa } \\
\text { en la cantidad de personas que demandan el } \\
\text { servicio. }\end{array}$ & $-2 \mid$ \\
\hline $\begin{array}{l}\text { Liquidez } \\
\text { El nivel de liquidez para demandas } \\
\text { inesperadas de caja ha tenido un } \\
\text { aumento del } 2 \%\end{array}$ & 3 & $\begin{array}{l}\text { Presión competitiva } \\
\text { La cooperativa de transporte urbano Las } \\
\text { Palmas ha adoptado nuevos métodos de } \\
\text { captar usuarios como, nuevos horarios, } \\
\text { nuevas unidades entre otras. }\end{array}$ & -3 \\
\hline $\begin{array}{l}\text { Capital de trabajo } \\
\text { El porcentaje de capital de trabajo está } \\
\text { por debajo del requerido que es de } 7 \%\end{array}$ & 1 & $\begin{array}{l}\text { Cambios tecnológicos } \\
\text { Nuevos herramientas tecnologías para conteo } \\
\text { de pasajeros ya a la venta en el país. }\end{array}$ & -1 \\
\hline Ventaja Competitiva (VC) & Valor & Fuerza de la Industria (FI) & Valor \\
\hline $\begin{array}{l}\text { Calidad del producto } \\
\text { La compañia mediante sus operarios, } \\
\text { ofrece un servicio apropiado en la mayoría } \\
\text { de las ocasiones }\end{array}$ & -3 & $\begin{array}{l}\text { Potencial de crecimiento } \\
\text { Aumento de la población, más la llegada de } \\
\text { migrantes que usan el transporte público } \\
\text { sugiere un ligero aumento de la demanda. }\end{array}$ & 2 \\
\hline $\begin{array}{l}\text { Participación en el mercado } \\
\text { Del } 100 \% \text { de la ciudadanía esmeraldeña } \\
\text { que se transporta en los buses, el } 50 \% \text { usa } \\
\text { los buses e la nuestra compañia. }\end{array}$ & -3 & $\begin{array}{l}\text { Estabilidad financiera } \\
\text { La taza de inflación tuvo un aumento del } \\
0.01 \% \text { entre el } 2017-2018\end{array}$ & 2 \\
\hline $\begin{array}{l}\text { Control sobre proveedores } \\
\text { El control sobre los proveedores es } \\
\text { moderado, pues existen varios que ofrecen } \\
\text { productos y servicios similares. }\end{array}$ & -3 & $\begin{array}{l}\text { Accesibilidad al mercado } \\
\text { Dado el tamaño de la ciudad de esmeraldas, } \\
\text { existe la baja posibilidad de que se desarrollen } \\
\text { nuevas cooperativas de transporte urbano }\end{array}$ & 4 \\
\hline
\end{tabular}

Tabla 5 Matriz de posición estratégica y evaluación de la acción (PEYEA)

La matriz PEYEA (Tabla 5) indica según los factores tomados en cuenta que afectan a la empresa, que esta debe tomar como opción alternativa la elección de estrategias conservadoras que le permitan mantenerse en el mercado y posteriormente aumentar su nivel competitivo.

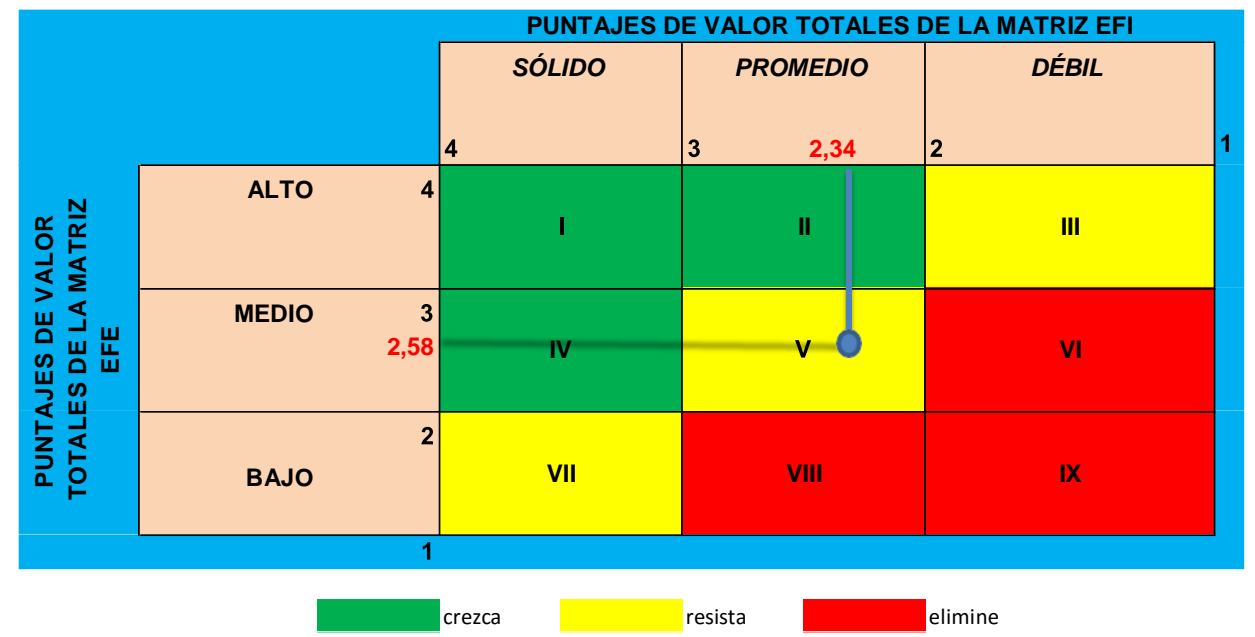

Tabla 6 Matriz interna-externa (IE) 
La matriz IE (Tabla 6) muestra que la empresa se encuentra en el cuadrante V es decir conservar y mantener, esto indica que la cooperativa San Jacinto debe desarrollar y aplicar estrategias de penetración en el mercado y desarrollo de nuevos productos, o en este caso, el servicio de rutas que ofrece.

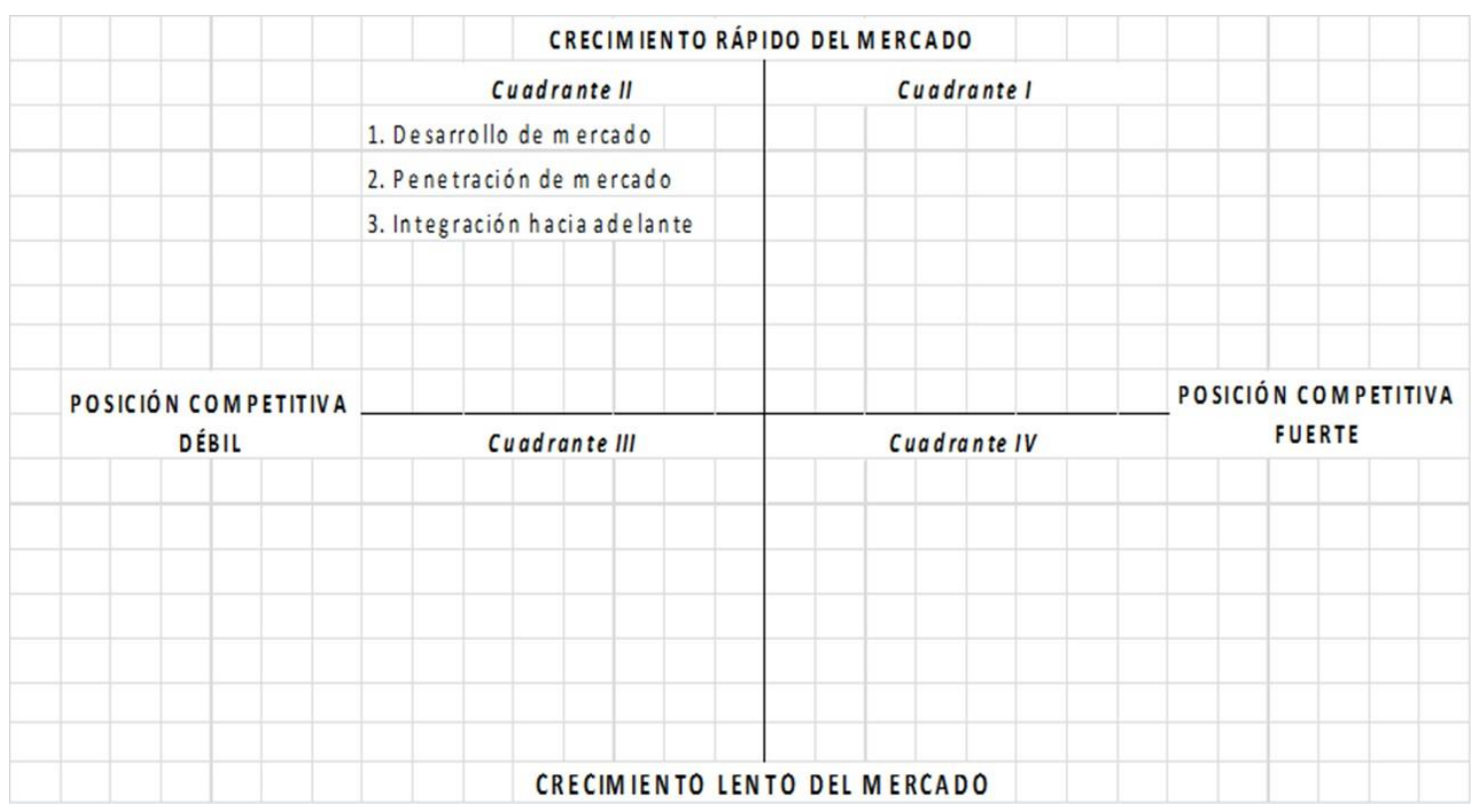

Tabla 7 Matriz de la estrategia principal

La matriz de la estrategia principal (Tabla 7) nos indica que la cooperativa se encuentra en condiciones favorables en cuanto al crecimiento del mercado, pero debe implementar estrategias que le permitan explotar los puntos positivos, lo que implica aumentar su nivel de competitividad.

La matriz MCPE (Tabla 8) muestra que la estrategia de Realizar petición formal de acceso a nuevas rutas tiene una calificación de 2,46 lo que significa que es más atractiva que la estrategia de reestructuración de los horarios de la ruta, según la hora de inicio y culminación de las jornadas estudiantiles con una calificación de 2,13. Esto sugiere que es mejor optar por la primera estrategia mencionada. 


\begin{tabular}{|c|c|c|c|c|c|}
\hline \multicolumn{2}{|l|}{ Factores criticos del exito } & \multicolumn{2}{|c|}{$\begin{array}{l}\text { Realizar petición formal de } \\
\text { acceso a nuevas rutas. }\end{array}$} & \multicolumn{2}{|c|}{$\begin{array}{c}\text { Reestructuración de los } \\
\text { horarios de la ruta, según la } \\
\text { hora de inicio y culminación } \\
\text { de las jornadas }\end{array}$} \\
\hline Oportunidades & Peso & CA & TCA & CA & TCA \\
\hline O1. Financiamiento externo para la renovación de oficinas administrativas & 0,05 & - & & - & \\
\hline O2. Incremento de estudiantes que demandan transporte urbano & 0,06 & 3 & 0,18 & 4 & 0,24 \\
\hline O3. Desarrollo de nuevas tecnología para el control de actividades & 0,05 & _- & & _- & _- \\
\hline O4. Mejoramiento en vías de circulación dentro del cantón & 0,06 & 4 & 0,24 & 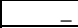 & \\
\hline O5. Demanda insatisfecha en la ruta correspondiente a la competencia & 0,07 & 4 & 0,28 & 3 & 0,18 \\
\hline O6. Seminarios gratuitos & 0,07 & _- & & _. & \\
\hline O7. Posibilidad de ampliar el perímetro permitido para el recorrido. & 0,07 & 3 & 0,21 & 3 & 0,21 \\
\hline O8. Desarrollo de nuevos métodos de control de recorridos & 0,05 & 1 & 0,05 & 1 & 0,05 \\
\hline \multicolumn{6}{|l|}{ Amenaza } \\
\hline A1. Posible aumento en el costo de combustible & 0,07 & - & _- & $=$ & - \\
\hline A2. Aumento de transporte informal & 0,07 & 3 & 0,21 & 3 & 0,21 \\
\hline A3. Atraso en el desarrollo económico de la Provincia & 0,07 & _- & - & $=$ & - \\
\hline A4. Aumento en el costo de mantenimiento y de implementos de las unidades. & 0,06 & _- & _- & - & _- \\
\hline A5. Competencia obtiene nuevo acuerdo para ruta universitaria. & 0,05 & 4 & 0,2 & 3 & 0,15 \\
\hline A6. Aumento de la inseguridad social & 0,06 & 2 & 0,12 & 3 & 0,18 \\
\hline A7. Presentación de nuevas unidades de transporte en la competencia. & 0,05 & _ & - & - & $=$ \\
\hline A8. Constantes cierres de carretera por reparación de tuberías. & 0,06 & 3 & 0,18 & 2 & 0,12 \\
\hline \multicolumn{6}{|l|}{ Fortalezas } \\
\hline F1. Funcionamiento administrativo en oficinas propias & 0,07 & _- & _ & - & _ \\
\hline F2. Personal operativo con licencia y demás documentación en regla & 0,06 & 3 & 0,18 & 3 & 0,18 \\
\hline F3. Cooperativa legalmente constituida & 0,06 & 3 & 0,18 & 2 & 0,12 \\
\hline F4. Personal con predisposición para cambios y mejoras & 0,06 & _- & _- & - & _ \\
\hline F5. Buena relación con proveedores & 0,07 & 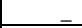 & & $=$ & - \\
\hline F6. Cantidad de unidades de flota suficientes para la demanda & 0,06 & 3 & 0,18 & 3 & 0,18 \\
\hline F7. Gerencia con experiencia en este tipo de negocio. & 0,06 & 2 & 0,12 & 3 & 0,18 \\
\hline F8. Buenas relaciones laborales. & 0,06 & _ & _ & - & _ \\
\hline \multicolumn{6}{|l|}{ Debilidades } \\
\hline D1. Ausencia de organigrama estructural. & 0,06 & _- & _- & - & _- \\
\hline D2. Dirigencia de la cooperativa con conocimiento administrativo limitado. & 0,06 & 1 & 0,06 & 1 & 0,06 \\
\hline D3. Inexistencia de sistemas de información empresarial apropiados & 0,06 & _- & - & - & _- \\
\hline D4. Inexistencia de reglamento interno & 0,06 & - & - & $=$ & - \\
\hline D5. Inexistencia de políticas y valores & 0,06 & _- & & - & _ \\
\hline D6. Actuales oficinas administrativas con espacio reducido & 0,06 & $t$ & _. & - & \\
\hline D7. Sistema de gestión y control de actividades obsoleto & 0,07 & 1 & 0,07 & 1 & 0,07 \\
\hline D8. Poca coordinación de actividades entre los trabajadores & 0,06 & _. & - & 1 & \\
\hline Total & & & 2,46 & & 2,13 \\
\hline
\end{tabular}

\section{Tabla 8 La matriz Cuantitativa De Planificación Estratégica (MCPE)}

\begin{tabular}{|c|c|c|c|c|c|c|}
\hline $\mathbf{N}$ & $\begin{array}{l}\text { PLAN } \\
\text { DE ACCIÓN } \\
\text { OBJETIVOS }\end{array}$ & $\begin{array}{l}\text { Actividad 1er } \\
\text { año }\end{array}$ & Actividad 2do año & Actividad 3er año & Actividad 4to año & RECURSOS \\
\hline 1 & $\begin{array}{l}\text { Fortalecer el } \\
\text { capital } \\
\text { humano }\end{array}$ & $\begin{array}{l}\text { Programar } \\
\text { cursos de } \\
\text { atención al } \\
\text { cliente y } \\
\text { relaciones } \\
\text { humanas }\end{array}$ & $\begin{array}{l}\text { Capacitaciones } \\
\text { en } \\
\text { mantenimiento } \\
\text { de vehículos }\end{array}$ & $\begin{array}{l}\text { Seminarios } \\
\text { sobre manejo } \\
\text { de equipos de } \\
\text { comunicación }\end{array}$ & $\begin{array}{l}\text { Capacitación } \\
\text { sobre } \\
\text { motivación y } \\
\text { autoestima }\end{array}$ & $\begin{array}{l}\text { Económicos- } \\
\text { Presupuestables }\end{array}$ \\
\hline 2 & $\begin{array}{l}\text { Adquirir } \\
\text { nuevas } \\
\text { unidades }\end{array}$ & $\begin{array}{l}\text { Mantener } \\
\text { actualizadas } \\
\text { cotizaciones } \\
\text { de nuevas } \\
\text { unidades }\end{array}$ & $\begin{array}{l}\text { Reuniones } \\
\text { mensuales para } \\
\text { determinar } \\
\text { proveedores }\end{array}$ & $\begin{array}{l}\text { Tramitar } \\
\text { financiamiento } \\
\text { externo }\end{array}$ & $\begin{array}{l}\text { Renovación } \\
\text { gradual de la } \\
\text { totalidad de las } \\
\text { unidades }\end{array}$ & Finanzas \\
\hline 3 & $\begin{array}{l}\text { Reducir el } \\
\text { tiempo de } \\
\text { marcaje de } \\
\text { tarjetas de } \\
\text { las unidades }\end{array}$ & $\begin{array}{l}\text { Implementar } \\
\text { procedimient } \\
\text { os para } \\
\text { marcaje de } \\
\text { tarjetas }\end{array}$ & $\begin{array}{l}\text { Adquisición de } \\
\text { sistema } \\
\text { automático } \\
\text { registro de } \\
\text { tarjetas }\end{array}$ & $\begin{array}{l}\text { Uso de los } \\
\text { sistemas de } \\
\text { comunicación } \\
\text { para los } \\
\text { marcadores }\end{array}$ & $\begin{array}{l}\text { Programación } \\
\text { de rutas y } \\
\text { tempos para } \\
\text { arribo de buses } \\
\text { a marcadores }\end{array}$ & Económicos \\
\hline 4 & $\begin{array}{l}\text { Incorporar } \\
\text { nuevos } \\
\text { socios a la } \\
\text { cooperativa }\end{array}$ & $\begin{array}{l}\text { Modificación } \\
\text { de valor de } \\
\text { acciones }\end{array}$ & $\begin{array}{l}\text { Campaña para } \\
\text { reclutamiento } \\
\text { de nuevos } \\
\text { socios }\end{array}$ & $\begin{array}{l}\text { Ventas de } \\
\text { nuevas } \\
\text { acciones }\end{array}$ & $\begin{array}{l}\text { Promociones de } \\
\text { nuevas } \\
\text { acciones }\end{array}$ & $\begin{array}{l}\text { Talento humano- } \\
\text { económicos }\end{array}$ \\
\hline
\end{tabular}

Pol. Con. (Edición núm. 28) Vol. 3, No 12, diciembre 2018, pp. 63-84, ISSN: 2550 - 682X 


\section{Conclusiones}

Una vez terminado el trabajo realizado a la Cooperativa de Transporte Urbano San Jacinto S.A., se tiene claro el panorama de lo que se puede realizar con la implementación de un Plan Estratégico, por lo que se hace necesario cambiar la gestión administrativa de la misma.

Se evidenció una gran predisposición de parte de los administrativos y operativos para participar en las diferentes sesiones de trabajo efectuadas para la elaboración del diagnóstico situacional y la elaboración participativa de la misión, visión, objetivos estratégicos.

Los directivos tienen poco conocimiento del cuerpo legal que los rige, y de conocimientos relacionados a las actividades de planificación, en cierta forma es una de las causas del temor a los cambios o innovaciones, debido a que como todo ser humano teme siempre a lo desconocido.

\section{Referencias Bibliográficas}

García-Tenorio Ronda, J., \& Pérez Rodríguez, M. J. (1996). La misión corporativa: Una Declaración de Propósitos Estable. Cuadernos de estudios empresariales (6).

Méndez, D. P. (2015). APLICACIÓN DEL MODELO DE LAS 7S DE MCKINSEY Retrieved noviembre, 2018, from http://repository.udistrital.edu.co/bitstream/11349/7335/1/Diana\%20Paola\%20Mendez\%2 02015.pdf

Murillo, D., \& Chasiluisa, C. (2012). PLANIFICACIÓN ESTRATÉGICA APLICADA A LA EMPRESA. $\quad$ Retrieved noviembre, 2018, from http://dspace.ucuenca.edu.ec/bitstream/123456789/1104/1/tad1100.pdf

Peiro Ucha, A. (2018). Visión de una empresa. from https://economipedia.com/definiciones/vision-de-una-empresa.html

Riquelme Leiva, M. (2015). Las 5 Fuerzas de Porter - Clave para el Éxito de la Empresa. Retrieved noviembre, 2018, from http://www.5fuerzasdeporter.com/

Riquelme, M. (2018). La cadena de valor de Michael Porter. Retrieved noviembre, 2018, from https://www.webyempresas.com/la-cadena-de-valor-de-michael-porter/ 